\title{
The Cause of Death of a Child in the 18th Century Solved by Bone Microbiome Typing Using Laser Microdissection and Next Generation Sequencing
}

\author{
Valeria D'Argenio ${ }^{1,2}$, Marielva Torino ${ }^{3}$, Vincenza Precone ${ }^{1}$, Giorgio Casaburi ${ }^{1,+}$, \\ Maria Valeria Esposito ${ }^{1}$, Laura Iaffaldano ${ }^{1}$, Umberto Malapelle ${ }^{4}$, Giancarlo Troncone ${ }^{4}$, \\ Iolanda Coto ${ }^{1,2}$, Paolina Cavalcanti ${ }^{5}$, Gaetano De Rosa ${ }^{6}$, Francesco Salvatore ${ }^{1,7, *}$ \\ and Lucia Sacchetti ${ }^{1 \text {,* }}$ \\ 1 CEINGE-Biotecnologie Avanzate, via G. Salvatore 486, 80145 Naples, Italy; \\ dargenio@ceinge.unina.it (V.D.); precone@ceinge.unina.it (V.P.); giorgio.casaburi@gmail.com (G.C.); \\ espositomari@ceinge.unina.it (M.V.E.); iaffaldano@ceinge.unina.it (L.I.); iolanda.coto@unina.it (I.C.) \\ Department of Molecular Medicine and Medical Biotechnologies, University of Naples Federico II, \\ via Pansini 5, 80131 Naples, Italy \\ 3 Laboratory of Archeo-Anthropology, University of Naples Suor Orsola Benincasa, \\ via Suor Orsola 10, 80125 Naples, Italy; marielvatorino@libero.it \\ 4 Department of Public Health, University of Naples Federico II, Naples, via Pansini 5, 80131 Naples, Italy; \\ umberto.malapelle@unina.it (U.M.); giancarlo.troncone@unina.it (G.T.) \\ 5 Microbiology Unit, Hospital of Cosenza, via San Martino, 87100 Cosenza, Italy; batteriologia@libero.it \\ 6 Department of Advanced Biomedical Sciences, University of Naples Federico II, \\ via Pansini 5, 80131 Naples, Italy; gaderosa@unina.it \\ 7 IRCCS (Istituto di Ricovero e Cura a Carattere Scientifico)-Fondazione SDN, \\ via Gianturco 113, 80143 Naples, Italy \\ * Correspondence: salvator@unina.it (F.S.); sacchett@unina.it (L.S.); Tel.: +39-081-746-3648 (F.S.); \\ +39-081-373-7827 (L.S.); Fax+39-081-746-3133 (F.S.); +39-081-373-7808 (L.S.) \\ + Present address: Life Sciences Lab, University of Florida, Kennedy Space Center, \\ Merritt Island, FL 32953, USA.
}

Academic Editor: William Chi-shing Cho

Received: 28 October 2016; Accepted: 3 January 2017; Published: 6 January 2017

\begin{abstract}
The history of medicine abounds in cases of mysterious deaths, especially by infectious diseases, which were probably unresolved because of the lack of knowledge and of appropriate technology. The aim of this study was to exploit contemporary technologies to try to identify the cause of death of a young boy who died from a putative "infection" at the end of the 18th century, and for whom an extraordinarily well-preserved minute bone fragment was available. After confirming the nature of the sample, we used laser microdissection to select the most "informative" area to be examined. Tissue genotyping indicated male gender, thereby confirming the notary's report. $16 \mathrm{~S}$ ribosomal RNA sequencing showed that Proteobacteria and Actinobacteria were more abundant than Firmicutes and Bacteroidetes, and that Pseudomonas was the most abundant bacterial genus in the Pseudomonadaceae family. These data suggest that the patient most likely died from Pseudomonas osteomyelitis. This case is an example of how new technological approaches, like laser microdissection and next-generation sequencing, can resolve ancient cases of uncertain etiopathology. Lastly, medical samples may contain a wealth of information that may not be accessible until more sophisticated technology becomes available. Therefore, one may envisage the possibility of systematically storing medical samples for evaluation by future generations.
\end{abstract}

Keywords: metagenomics; human microbiome; cold case; next generation sequencing 


\section{Introduction}

Past cases of death from unknown causes, particularly those of famous personalities, are commonly referred to as "mysterious deaths". In many instances, such cases can now be solved thanks to advances in research and to the advent of modern technologies [1,2]. Notably, identification of causes of death in ancient "cold cases" may also shed light on the evolution of a disease [3]. This article concerns a young boy who died at the end of the 18th century from a putative infection of a bone wound (possibly osteomyelitis). His parents felt that their son was not receiving appropriate treatment because his condition was not correctly diagnosed. Consequently, they took the unusual step of preserving a fragment of the affected bone for future analysis. Thus, the fragment was sealed by a public notary in a small envelope (see the documentation in the Supplementary File 1 and Figure S1) until the study described in this paper.

Next-generation technology for high-throughput sequencing of nucleic acids has been a breakthrough in the field of metagenomics, and has produced a wealth of data about various environmental niches in medical research, particularly in the field of infectious diseases $[4,5]$. The aim of our study was to try to identify the cause of death of this boy using contemporary technologies and methods.

\section{Results}

Hematoxylin and eosin ( $\mathrm{H}$ and $\mathrm{E})$ stained sections of the specimen showed the features typical of bone tissue and aggregates of granulocytes, which is indicative of an inflammatory reaction (Figure 1A). To minimize the risk of external contamination and obtain a sufficient quantity of DNA-rich material, we isolated a piece of the infected bone by laser microdissection under sterile conditions. Figure 1B shows the pre-microdissected area, and Figure 1C the post-microdissected area. Sex-determining region Y (SRY) analysis confirmed that the DNA was from a male (Figure 1D).

A

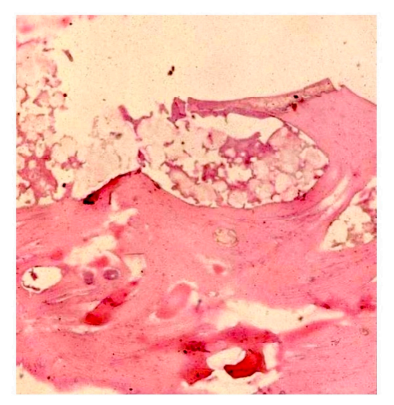

C

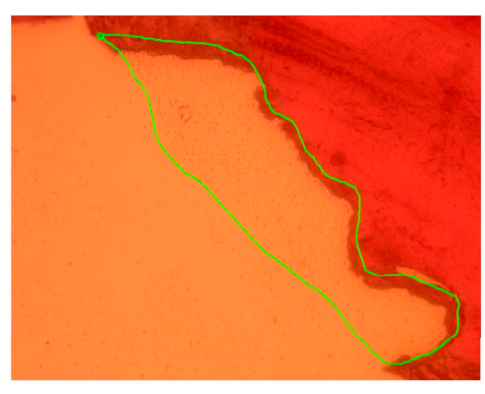

B

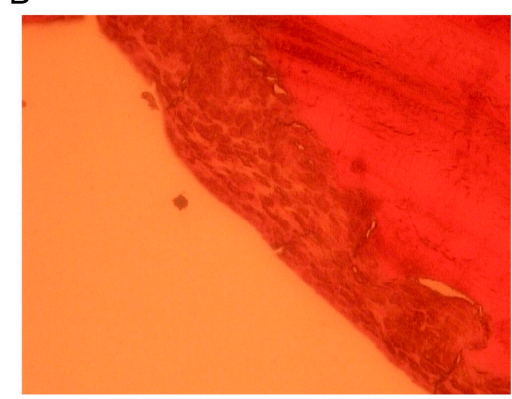

D $\underset{100-1200 \text { bp }}{\text { Ladder }} \underset{\text { Negative }}{\text { Nontrol }}$ Male controls $\quad$ Uncharged slots

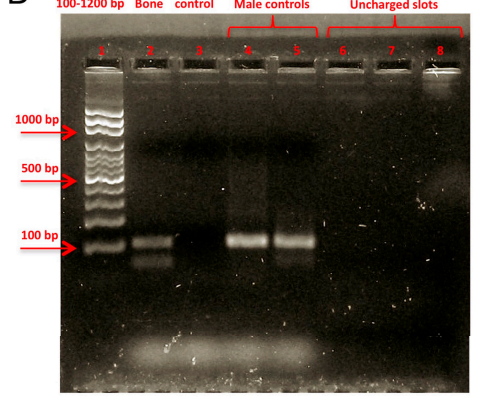

Figure 1. Bone sample characterization. (A) Microscopy image (hematoxylin and eosin staining) showing the features typical of bone tissue and inflammatory infiltration; example of a laser captured microdissected area of the bone sample: (B) shows the pre-microdissected area $(40 \times$ resolution); (C) the green line encircles the microdissected area; (D) sex-determining region $\mathrm{Y}$ amplification revealed the sex of the patient: lane 1: ladder (100-1200 bp); lane 2: sample; lane 3: negative control; lanes 4 and 5: male controls; lanes 6-8: uncharged slots. 
We analyzed the entire bacterial microbiome of the bone sample by a next-generation sequencing approach, as described under Methods (see the Section 4.5). A total of 7,357,569 base pairs (bp) corresponding to $14,17216 \mathrm{~S}$ sequences (mean sequence length, $528.7 \mathrm{bp}$ and average quality score 34.18 ) were generated. After pre-quality filtering (see Section 4.6 for details), 12,829 high quality sequences were considered for subsequent analysis. In detail, 243 representative operational taxonomic units (OTUs) were obtained using an open-reference OTU picking approach [6]. The taxonomic assignment of these OTUs revealed four bacterial phyla. As shown in Figure 2A, the most abundant phylum was Proteobacteria (59\%) followed by Actinobacteria and Firmicutes (16\% each) and Bacteroidetes $(9 \%)$. A low percent of OTUs $(0.2 \%)$ was unclassified (data not shown). The bacterial distribution at the class level (Figure 2B) show the prevalence of Gammaproteobacteria, Bacilli and Actinobacteria (at a frequency of $45 \%, 16 \%$ and $15 \%$, respectively). At Order level (Figure 2C), six orders were most abundant: Pseudomanadales (16\%), Actinomycetales (15\%), Lactobacillales (14\%) Enterobacteriales $(11 \%)$, Alteromonadales (10\%), and Sphingobacteriales (8\%). Figure 2D shows the main bacterial families, six of which had more than 1000 assigned sequences: Sphingobacteriaceae $(9 \%)$, Pseudonocardiaceae (about 10\%), Alteromonadaceae (11\%), Enterobacteriaceae (12\%), Leuconostocaceae $(14 \%)$, and Pseudomonadaceae (15\%). Interestingly, as shown in Figure 2E, Pseudomonadaceae presents the most abundant bacteria at genus level (i.e., Pseudomonas; $15 \%$ ).

B

A

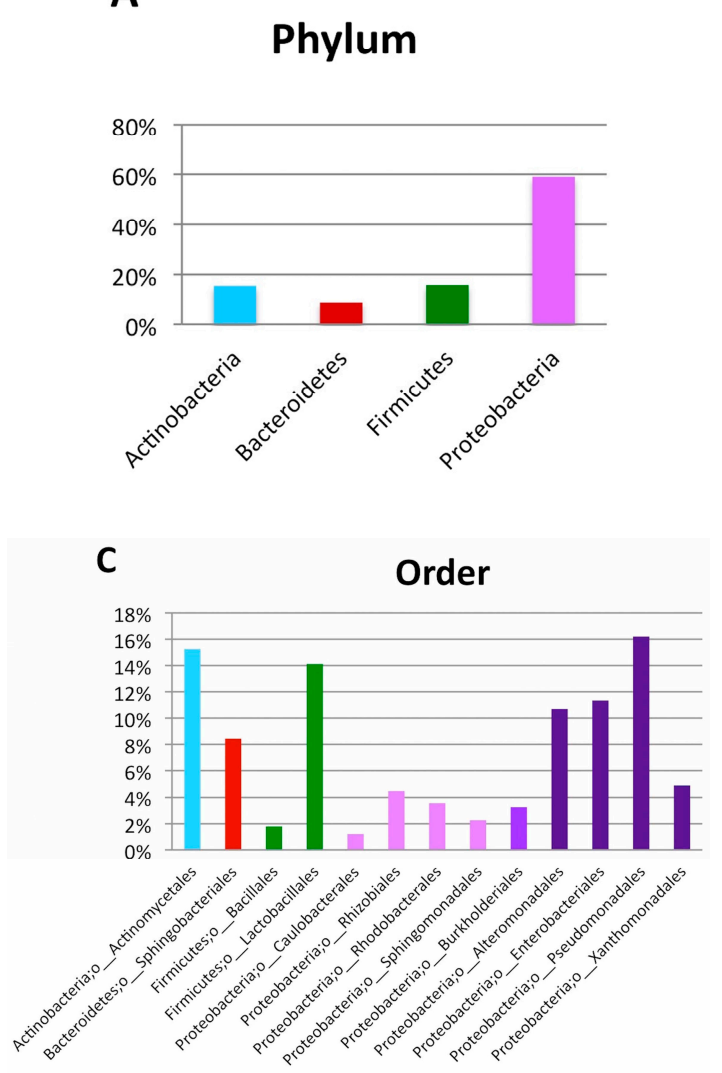

\section{Class}

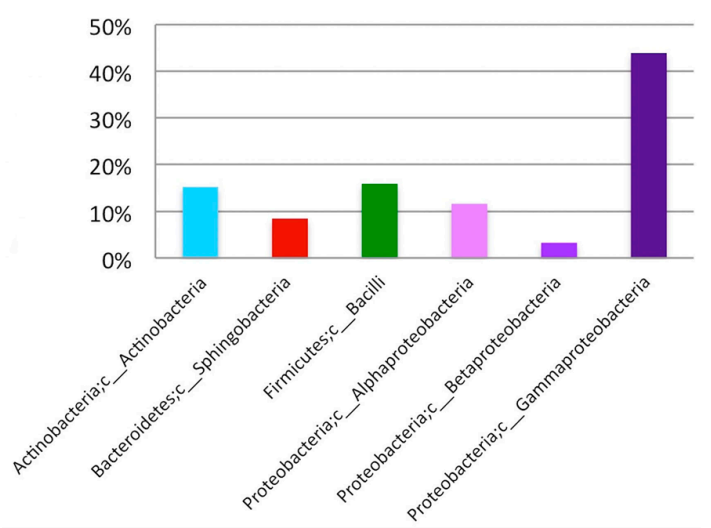

D

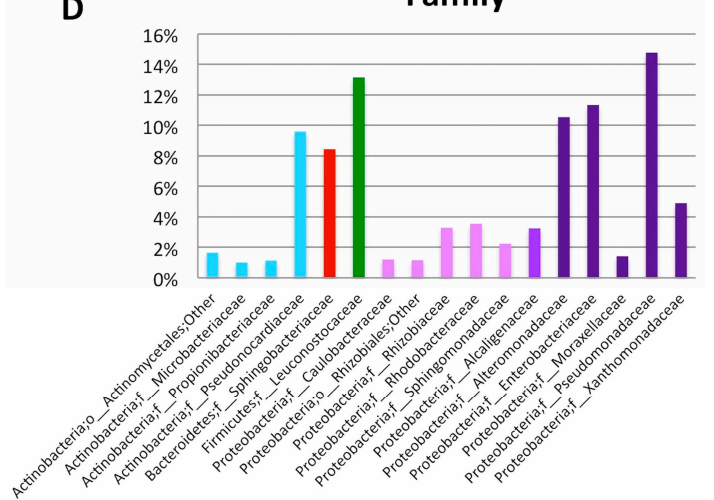

Figure 2. Cont. 


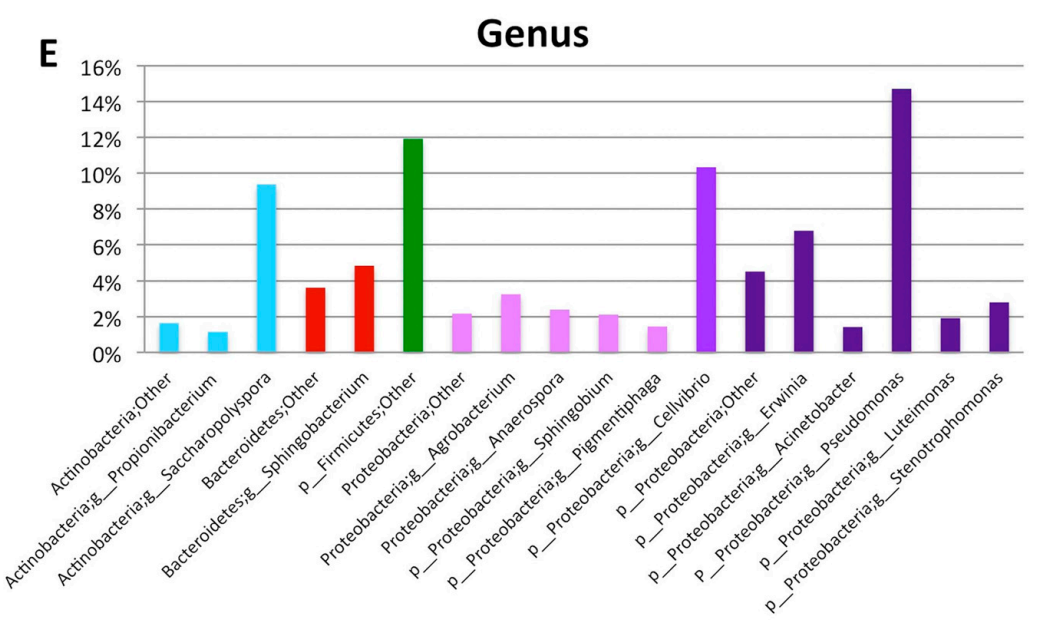

Figure 2. Bone microbiome composition. (A-E) 16S rRNA sequencing of the bone tissue microbiome. Bacterial composition (frequency $>1 \%$ ) is shown from phylum to genus level. (A) Proteobacteria was the most abundant phylum with a frequency of $59 \%$; within this phylum, we found the prevalence of Gammaproteobacteria (45\% of frequency, class level, panel B); Pseudomonadales (16\% of frequency, order level, panel C); Pseudomonadaceae (15\% of frequency, family level, panel D); and Pseudomonas (15\% of frequency, genus level, panel E).

A complete list of the bacteria that reached the deepest taxonomic assignment (i.e., genus) using a filter of $0.05 \%$ of OTU frequency is shown in Table 1 and Supplementary Figure S2.

\section{Discussion}

Osteomyelitis has long been known, but the cause of this infection remained obscure until the advent of methods able to identify disease-causing bacterial species. For instance, $P$. aeruginosa was discovered only in 1882. This bacterium is present in diverse chronic severe infectious diseases and is also one of the most difficult to eradicate, a notable case being cystic fibrosis [7,8]. P. aeruginosa has been also reported as causative agent of osteomyelitis [9].

We had the unusual opportunity of evaluating with contemporary techniques a well-preserved bone fragment from a child who died in the 18th century from a putative infection. The abundance of the genus Pseudomonas and the semi-chronic nature of the infection in the case reported (see Supplementary Materials) strongly suggest that the cause of death was an infection by this bacterial genus. This diagnosis is supported by the whole dataset analyzed. In fact, after assessing the nature of the recovered ancient bone fragment, the analysis of the entire microbiome composition of the bone showed that Pseudomonas (within the Pseudomonadaceae family) was the most abundant genus identified (15\%), which suggests it was the disease-etiopathogenetic agent of our "cold case" [10].

Environmental contamination cannot be excluded in such an ancient bone sample. However, we detected low abundance of genera typically found in soil or plants (e.g., Agrobacterium and Janthinobacterium, see Table 1); consequently, we exclude that their presence could affect the result relative to the putative pathogenic role of Pseudomonas, which was highly present in our sample. Further, among the most abundant genera, Pseudomonas, Sphingobacterium, and Cellvibrio, only the first one includes species which are highly pathogenic [8,10-12]. In line with our hypothesis, Pseudomonas was recently identified in five patients affected by diabetic foot osteomyelitis [13]. In the latter study, 16S rRNA sequencing showed that Pseudomonas constituted $18 \%$ to the total bacterial population, similar to the $15 \%$ observed by us.

The rare presence of this lineage in our sample suggests that the possibility of analyzing antique remains of biological samples is certainly useful to be approached by micro-environment microbiome typing. Lastly, the relevant authorities could consider the feasibility of setting up archives of "difficult-to-diagnose" biological material for future evaluation. 
Table 1. List of all of the bacteria identified at the deepest reachable taxonomic level in the bone sample by 16S rRNA next-generation sequencing. The bacteria are reported according to their relative abundance (at genus level) without considering OTUs that have not been assigned to the genus level. For each of them, the taxonomic assignment at five phylogenetic levels (from phylum to genus), the relative abundances at genus level (the most abundant being $15 \%$ for Pseudomonas), and the number of high-quality mapping reads are reported at the genus level. Total reads (post-quality OTU classifications) $=4244$. Part of the reads (about $11 \%$ ) are not assigned down to the genus level). Unidentified reads $=0.009 \%$.

\begin{tabular}{|c|c|c|c|c|c|c|}
\hline Phylum & Class & Order & Family & Genus & $\begin{array}{c}\text { Relative Abundance at } \\
\text { Genus Level (\%) }\end{array}$ & $\begin{array}{l}\text { Number of High Quality } \\
\text { Reads at Genus Level }\end{array}$ \\
\hline Actinobacteria & Actinobacteria & Actinomycetales & Brevibacteriaceae & Brevibacterium & 0.31 & 9 \\
\hline Actinobacteria & Actinobacteria & Actinomycetales & Corynebacteriaceae & Corynebacterium & 0.75 & 22 \\
\hline Actinobacteria & Actinobacteria & Actinomycetales & Microbacteriaceae & Agrococcus & 0.38 & 11 \\
\hline Actinobacteria & Actinobacteria & Actinomycetales & Microbacteriaceae & Leucobacter & 0.10 & 3 \\
\hline Actinobacteria & Actinobacteria & Actinomycetales & Microbacteriaceae & Microbacterium & 0.68 & 20 \\
\hline Actinobacteria & Actinobacteria & Actinomycetales & Micrococcaceae & Arthrobacter & 0.21 & 6 \\
\hline Actinobacteria & Actinobacteria & Actinomycetales & Micrococcaceae & Micrococcus & 0.96 & 28 \\
\hline Actinobacteria & Actinobacteria & Actinomycetales & Propionibacteriaceae & Propionibacterium & 1.34 & 39 \\
\hline Actinobacteria & Actinobacteria & Actinomycetales & Pseudonocardiaceae & Amycolatopsis & 5.14 & 150 \\
\hline Actinobacteria & Actinobacteria & Actinomycetales & Pseudonocardiaceae & Pseudonocardia & 1.13 & 33 \\
\hline Actinobacteria & Actinobacteria & Actinomycetales & Pseudonocardiaceae & Saccharopolyspora & 8.87 & 259 \\
\hline Bacteroidetes & Flavobacteriia & Flavobacteriales & Flavobacteriaceae & Flavobacterium & 0.41 & 12 \\
\hline Bacteroidetes & Sphingobacteriia & Sphingobacteriales & Sphingobacteriaceae & Sphingobacterium & 11.26 & 329 \\
\hline Firmicutes & Bacilli & Bacillales & Bacillaceae & Bacillus & 0.72 & 21 \\
\hline Firmicutes & Bacilli & Bacillales & Planococcaceae & Lysinibacillus & 0.14 & 4 \\
\hline Firmicutes & Bacilli & Bacillales & Planococcaceae & Rummeliibacillus & 0.79 & 23 \\
\hline Firmicutes & Bacilli & Bacillales & Staphylococcaceae & Staphylococcus & 0.45 & 13 \\
\hline Firmicutes & Bacilli & Lactobacillales & Aerococcaceae & Aerococcus & 0.79 & 23 \\
\hline Firmicutes & Bacilli & Lactobacillales & Enterococcaceae & Enterococcus & 0.07 & 2 \\
\hline Firmicutes & Bacilli & Lactobacillales & Streptococcaceae & Streptococcus & 0.45 & 13 \\
\hline Proteobacteria & Alphaproteobacteria & Caulobacterales & Caulobacteraceae & Brevundimonas & 0.10 & 3 \\
\hline Proteobacteria & Alphaproteobacteria & Rhizobiales & Rhizobiaceae & Agrobacterium & 4.86 & 142 \\
\hline Proteobacteria & Alphaproteobacteria & Rhodobacterales & Rhodobacteraceae & Anaerospora & 3.87 & 113 \\
\hline Proteobacteria & Alphaproteobacteria & Rhodobacterales & Rhodobacteraceae & Rhodobacter & 1.10 & 32 \\
\hline Proteobacteria & Alphaproteobacteria & Sphingomonadales & Sphingomonadaceae & Sphingobium & 3.22 & 94 \\
\hline Proteobacteria & Betaproteobacteria & Burkholderiales & Alcaligenaceae & Achromobacter & 0.27 & 8 \\
\hline Proteobacteria & Betaproteobacteria & Burkholderiales & Alcaligenaceae & Pigmentiphaga & 1.64 & 48 \\
\hline Proteobacteria & Betaproteobacteria & Burkholderiales & Oxalobacteraceae & Janthinobacterium & 0.10 & 3 \\
\hline Proteobacteria & Gammaproteobacteria & Alteromonadales & Alteromonadaceae & Cellvibrio & 10.50 & 482 \\
\hline Proteobacteria & Gammaproteobacteria & Enterobacteriales & Enterobacteriaceae & Erwinia & 4.59 & 134 \\
\hline Proteobacteria & Gammaproteobacteria & Pseudomonadales & Moraxellaceae & Acinetobacter & 1.92 & 56 \\
\hline Proteobacteria & Gammaproteobacteria & Pseudomonadales & Pseudomonadaceae & Pseudomonas & 15.00 & 584 \\
\hline Proteobacteria & Gammaproteobacteria & Xanthomonadales & Xanthomonadaceae & Luteimonas & 2.94 & 86 \\
\hline Proteobacteria & Gammaproteobacteria & Xanthomonadales & Xanthomonadaceae & Stenotrophomonas & 3.97 & 116 \\
\hline
\end{tabular}




\section{Materials and Methods}

\subsection{Sample Obtainment and Preparation for Analysis}

The bone fragment studied was contained in a sealed envelope deposited in the office of a Public Notary on 17 December 1792. On 5 May 1995, after obtaining authorization from the Ministry of Cultural Heritage (authorization number 2.2344), the tiny fragment of bone was extracted from the envelope under sterile conditions. The fragment was trapezoidal in shape, it measured $10 \mathrm{~mm} \times 15 \mathrm{~mm}$, and was white with jagged edges. This information is deposited in an official act of the National State Archives, Cosenza, Italy 5 May 1995. Now, the remaining bone fragment is deposited in the CEINGE-Biotecnologie Avanzate Bio-bank (2nd floor, lab B2/006, fridge block nr. 6). A very minute amount of DNA is still available.

\subsection{Hematoxylin and Eosin Staining}

$\mathrm{H}$ and $\mathrm{E} 5 \mu \mathrm{m}$ paraffin embedded sections were obtained as previously described [14].

\subsection{Laser Microdissection and DNA Extraction}

Using laser microdissection in combination with a laser pressure catapulting (PALM) system (MicroBeam, Microlaser Technologies $\mathrm{GmbH}$, Bernried, Germany), fixed cells were selectively dissected from $5-\mu \mathrm{m}$ paraffin-embedded $\mathrm{H}$ and $\mathrm{E}$ stained slides, under visual control according to the manufacturer's guidelines. Microdissected cells were collected in the adhesive cap of a 0.5-mL plastic tube (Carl Zeiss, Munich, Germany); $10 \mu \mathrm{L}$ of tissue lysis buffer A and $2 \mu \mathrm{L}$ of proteinase K (Qiagen, Crawley, West Sussex, UK) were added to the cells, which were then incubated for four hours at $56{ }^{\circ} \mathrm{C}$. After proteinase $\mathrm{K}$ inactivation, the DNA was purified using the QIAamp DNA Mini Kit (Qiagen) following the manufacturer's instructions. DNA quality was assessed by gel electrophoresis showing a high molecular weight band with a smear, thus ensuring that the DNA quality was sufficient for amplification and sequencing (Supplementary Figure S3A). To exclude external contamination, we also carried out the extraction procedure on the inside of the envelope well separated from the bone fragment, but no DNA was obtained, which indicated that no effective external contamination occurred (Supplementary Figure S3B).

\subsection{Sex-Determining Region Y Amplification}

PCR was carried out with $20 \mathrm{pmol}$ of each of the primers previously used by Shekhtman et al. [15], 1.69 nmol of each dNTP, $1 \times$ PCR HotMaster Taq Buffer (5 Prime, Milan, Italy), 0.5 U of HotMaster Taq (5 Prime), and $2 \mu \mathrm{L}$ of template DNA solution in a total volume of $25 \mu \mathrm{L}$. PCR conditions were as follows: initial denaturing at $94{ }^{\circ} \mathrm{C}$ for $2 \mathrm{~min} ; 35$ cycles of $94^{\circ} \mathrm{C}$ for $30 \mathrm{~s}, 55^{\circ} \mathrm{C}$ for $30 \mathrm{~s}, 65^{\circ} \mathrm{C}$ for $30 \mathrm{~s}$; and a final extension at $72{ }^{\circ} \mathrm{C}$ for $2 \mathrm{~min}$. Appropriate positive and negative controls were included. The PCR product underwent electrophoresis in agarose gel at $100 \mathrm{~V}$ for $30 \mathrm{~min}$.

\subsection{Microbiome Typing by $16 S$ rRNA Deep Sequencing}

A next-generation sequencing-based approach was used to investigate the microbiome of the bone fragment through deep 16S amplicon analysis. In detail, an aliquot of the extracted DNA was used for PCR amplification and sequencing of the bacterial $16 \mathrm{~S}$ gene. To investigate the bacterial composition of the bone sample, a 548 bp amplicon, spanning from the V4 to V6 variable regions of the 16S rRNA gene was amplified using the 519F (CAGCAGCCGCGGTAATAC) and the 1067R (TGACGACAGCCATGC) primers [16]. Both primers were modified to obtain fusion primers, each one containing at its $5^{\prime}$ end a universal 454 adaptor (adaptor A for the forward primer and adaptor B for the reverse one). The PCR reaction was carried out with $25 \mu \mathrm{L}$ of $\mathrm{H}_{2} \mathrm{O}, 20 \mu \mathrm{L}$ of $2.5 \times$ HotMaster PCR mix (Eppendorf, Hamburg, Germany), $1.5 \mu \mathrm{L}$ of each primer at $10 \mu \mathrm{M}$, and $60 \mathrm{ng}$ of DNA. The amplification was performed on a DNA ENGINE Chassis (Bio-Rad Laboratories, Hercules, CA, USA) under the following conditions: 
2 min at $94{ }^{\circ} \mathrm{C}, 30$ cycles of $94{ }^{\circ} \mathrm{C}$ for $40 \mathrm{~s}, 50{ }^{\circ} \mathrm{C}$ for $40 \mathrm{~s}$, and $65^{\circ} \mathrm{C}$ for $40 \mathrm{~s}$, and a final extension of $70{ }^{\circ} \mathrm{C}$ for $7 \mathrm{~min}$. Appropriate positive and negative controls were also amplified. In particular, to exclude possible contaminations, in addition to the bone DNA, we also carried out the 16S PCR amplicon preparation of the empty eluate obtained from the envelope and of a pure water control. Then, we assessed the presence of amplification on a $2 \%$ agarose gel stained with bromide ethidium: both the controls gave no amplification products, thus excluding contamination (Supplementary Figure S4). After visualization by gel electrophoresis, the bone PCR product was purified with Ampure magnetic purification beads (Agencourt Biosciences, Beverly, MA, USA), assessed for quality on a Bioanalyzer 2100 (Agilent Technologies, Santa Clara, CA, USA), and quantified using the Quant-it PicoGreen dsDNA kit (Invitrogen, Carlsband, CA, USA). This purified amplicon represents the next generation sequencing library to be sequenced on the 454 Genome Sequencer FLX instrument (Roche, Penzberg, Germany), following manufacturer's instructions as previously described [17].

\subsection{Bioinformatics}

16S amplicon sequences were analyzed using QIIME tool v. 1.9.1 (http:/ / qiime.org, Flagstaff, AZ, USA), which allows analysis of high-throughput microbial community sequencing data [18,19]. sff files were processed to extract FASTA sequences which were quality filtered using default parameters. In detail, sequences with: (i) length outside bounds $<200 \mathrm{nt}$ and $>1000 \mathrm{nt}$; (ii) a quality score $<25$; (iii) ambiguous bases; (iv) primer mismatches; and (v) homopolymer runs in excess of six bases, were removed. Clustering of OTUs was based on 97\% similarity using UCLUST tool (http:/ / drive5.com/ usearch/manual/uclust_algo.html, London, UK) [20], which was also chosen to assign taxonomy with a cutoff of $90 \%$ identity against the $16 \mathrm{~S}$ rDNA Greengenes database v. 13_08 [21]. Chimeric OTUs were removed using ChimeraSlayer tool (http://microbiomeutil.sourceforge.net/\#A_CS, Cambridge, MA, USA) [22].

\section{Conclusions}

Modern techniques, such as next generation sequencing for nucleic acid analysis, and laser microdissection to select the cells to be analyzed from the matrix of the mixed cells/tissues present in the sample, are now being used also to reveal the "cold cases" of ancient remains. These innovative techniques can reveal specific characteristics or the cause of mysterious deaths. In fact, in the case reported herein, a child of the 18th century died after a lengthy bone disease has now been found as affected by a bacterial strain not known at the time, the Pseudomonas genus. This paper might have interesting forensic aspects, and may prompt the preservation of samples of uncertain characteristics for future innovative technologies.

Supplementary Materials: Supplementary materials can be found at www.mdpi.com/1422-0067/18/1/109/s1.

Acknowledgments: This work has been supported by Grant PON03PE_00060_2 and PON03PE_00060_7 (Campania-Bioscience) from the Italian Ministry of University and Research (to Francesco Salvatore.). The authors thank: William Chi-Shing Cho, Academic editor of International Journal of Molecular Sciences for helpful comments which made significative improvements in the present paper; Lucia Chinigò, Archivist at the State Records Office of Cosenza, for the archive research; Anna Maria Letizia Fazio, Ex Director of the State Records Office of Cosenza, for authorizing this research; Jean Ann Gilder (Scientific Communication srl., Naples, Italy) for editing the text, Vittorio Lucignano, CEINGE-Biotecnologie Avanzate for technical assistance.

Author Contributions: Lucia Sacchetti, Francesco Salvatore, Valeria D'Argenio, and Marielva Torino conceived and designed the experiments; Valeria D'Argenio, Vincenza Precone, Giorgio Casaburi, Maria Valeria Esposito, Laura Iaffaldano, Umberto Malapelle, Giancarlo Troncone, Iolanda Coto, Paolina Cavalcanti and Gaetano De Rosa performed the experiments; Lucia Sacchetti, Francesco Salvatore, Valeria D'Argenio, Gaetano De Rosa and Marielva Torino analyzed the data; Lucia Sacchetti, Francesco Salvatore and Valeria D'Argenio wrote the paper. All authors read and approved the final manuscript.

Conflicts of Interest: The authors declare no conflict of interest. 


\section{Abbreviations}

H\&E Hematoxylin and eosin

SRY Sex-determining region $\mathrm{Y}$

OTUs Operational taxonomic units

\section{References}

1. Lugli, A.; Clemenza, M.; Corso, P.E.; di Costanzo, J.; Dirnhofer, R.; Fiorini, E.; Herborg, C.; Hindmarsh, J.T.; Orvini, E.; Piazzoli, A.; et al. The medical mystery of Napoleon Bonaparte: An interdisciplinary exposé. Adv. Anat. Pathol. 2011, 18, 152-158. [CrossRef] [PubMed]

2. Gallassi, F.M.; Ashrafian, H. Has the diagnosis of a stroke been overlooked in the symptoms of Julius Caesar? Neurol. Sci. 2015, 36, 1521-1522. [CrossRef] [PubMed]

3. Zink, A.R.; Reischl, U.; Wolf, H.; Nerlich, A.G. Molecular analysis of ancient microbial infections. FEMS Microbiol. Lett. 2002, 213, 141-147. [CrossRef] [PubMed]

4. Padmanabhan, R.; Mishra, A.K.; Raoult, D.; Fournier, P.E. Genomics and metagenomics in medical microbiology. J. Microbiol. Methods 2013, 95, 415-424. [CrossRef] [PubMed]

5. D'Argenio, V.; Salvatore, F. The role of the gut microbiome in the healthy adult status. Clin. Chim. Acta 2015, 451, 97-102. [CrossRef] [PubMed]

6. Edgar, R.C. Search and clustering orders of magnitude faster than BLAST. Bioinformatics 2010, 26, $2460-2461$. [CrossRef] [PubMed]

7. McCarthy, K. Pseudomonas aeruginosa: Evolution of antimicrobial resistance and implications for therapy. Semin. Respir. Crit. Care Med. 2015, 36, 44-55. [CrossRef] [PubMed]

8. Lenney, W. Pseudomonas aeruginosa in cystic fibrosis is potentially serious, and more than merely a marker for disease severity. Paediatr. Respir. Rev. 2015, 16, 35-36. [CrossRef] [PubMed]

9. Aydın, N.; Şirin, E.; Aydemir, A.N.; Zengin, G. Pseudomonas osteomyelitis of the proximal humerus after arthroscopic rotator cuff repair. Acta Orthop. Traumatol. Turc. 2014, 48, 685-689. [CrossRef] [PubMed]

10. Anzai, Y.; Kim, H.; Park, J.Y.; Wakabayashi, H.; Oyaizu, H. Phylogenetic affiliation of the pseudomonads based on 16S rRNA sequence. Int. J. Syst. Evol. Microbiol. 2000, 50, 1563-1589. [CrossRef] [PubMed]

11. Barahona, F.; Slim, J. Sphingobacterium multivorum: Case report and literature review. New Microbes New Infect. 2015, 7, 33-37. [CrossRef] [PubMed]

12. Gardner, J.G. Polysaccharide degradation systems of the saprophytic bacterium Cellvibrio japonicus. World J. Microbiol. Biotechnol. 2016, 32, 121. [CrossRef] [PubMed]

13. Van Asten, S.A.; La Fontaine, J.; Peters, E.J.; Bhavan, K.; Kim, P.J.; Lavery, L.A. The microbiome of diabetic foot osteomyelitis. Eur. J. Clin. Microbiol. Infect. Dis. 2016, 35, 293-298. [CrossRef] [PubMed]

14. Pasyk, K.A.; Hassett, C.A. Modified hematoxylin and eosin staining method for epoxy-embedded tissue sections. Pathol. Res. Pract. 1989, 184, 635-638. [CrossRef]

15. Shekhtman, E.M.; Anne, K.; Melkonyan, H.S.; Robbins, D.J.; Warsof, S.L.; Umansky, S.R. Optimization of transrenal DNA analysis: Detection of fetal DNA in maternal urine. Clin. Chem. 2009, 55, 723-729. [CrossRef] [PubMed]

16. Baker, G.C.; Smith, J.J.; Cowan, D.A. Review and re-analysis of domain-specific $16 \mathrm{~S}$ primers. J. Microbiol. Methods 2003, 55, 541-555. [CrossRef] [PubMed]

17. D'Argenio, V.; Casaburi, G.; Precone, V.; Pagliuca, C.; Colicchio, R.; Sarnataro, D.; Discepolo, V.; Kim, S.M.; Russo, I.; del Vecchio Blanco, G.; et al. Metagenomics Reveals Dysbiosis and a Potentially Pathogenic N. flavescens Strain in Duodenum of Adult Celiac Patients. Am. J. Gastroenterol. 2016, 111, 879-890. [CrossRef] [PubMed]

18. Caporaso, J.G.; Kuczynski, J.; Stombaugh, J.; Bittinger, K.; Bushman, F.D.; Costello, E.K.; Fierer, N.; Peña, A.G.; Goodrich, J.K.; Gordon, J.I.; et al. QIIME allows analysis of high-throughput community sequencing data. Nat. Methods 2010, 7, 335-336. [CrossRef] [PubMed]

19. D'Argenio, V.; Casaburi, G.; Precone, V.; Salvatore, F. Comparative metagenomic analysis of human gut microbiome composition using two different bioinformatic pipelines. BioMed Res. Int. 2014, 2014, 325340. [CrossRef] [PubMed] 
20. Wang, Q.; Garrity, G.M.; Tiedje, J.M.; Cole, J.R. Naive Bayesian Classifier for Rapid Assignment of rRNA Sequences into the New Bacterial Taxonomy. Appl. Environ. Microbiol. 2007, 73, 5261-5267. [CrossRef] [PubMed]

21. De Santis, T.Z.; Hugenholtz, P.; Larsen, N.; Rojas, M.; Brodie, E.L.; Keller, K.; Huber, T.; Dalevi, D.; Hu, P.; Andersen, G.L. Greengenes, a chimera-checked $16 \mathrm{~S}$ rRNA gene database and workbench compatible with ARB. Appl. Environ. Microbiol. 2006, 72, 5069-5072. [CrossRef] [PubMed]

22. Haas, B.J.; Gevers, D.; Earl, A.M.; Feldgarden, M.; Ward, D.V.; Giannoukos, G.; Ciulla, D.; Tabbaa, D.; Highlander, S.K.; Sodergren, E.; et al. Chimeric $16 \mathrm{~S}$ rRNA sequence formation and detection in Sanger and 454-pyrosequenced PCR amplicons. Genome Res. 2011, 21, 494-504. [CrossRef] [PubMed]

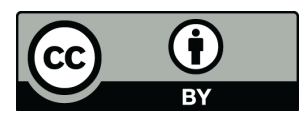

(C) 2017 by the authors; licensee MDPI, Basel, Switzerland. This article is an open access article distributed under the terms and conditions of the Creative Commons Attribution (CC-BY) license (http://creativecommons.org/licenses/by/4.0/). 